\title{
CPD QUESTIONS
}

Journal 41

Two CPD points are awarded for the correct completion and submission of the questions below.

CPD questionnaires must be completed online via www.cpdjournals.org.za.

After submission you can check the answers and print your certificate.

Questions may be answered up to 6 months after publication of each issue.

This programme is available free of charge to members of the HIV Clinicians Society and SAMA only.

Regarding the use of antiretroviral therapy to prevent the transmission of HIV in serodiscordant partnerships:

1. True (A) or false (B):

Observational studies have suggested that the lower the average viral load in a community, the lower the incidence of new HIV infections.

2. True (A) or false (B):

The HPTN 052 trial provides evidence that initiation of ART at a threshold of 350 - 550 cells/ $/$ l reduces sexual transmission of HIV to HIV-negative partners.

3. True (A) or false (B):

The HPTN 052 trial provides clear evidence that initiation of ART at a threshold of 350 - 550 cells/ $\mu$ reduces mortality and AIDS-related complications in HIV-infected individuals.

4. Evidence for the benefits of ART initiation above 500 cells/ $\mu$ is mixed, and trials investigating these are ongoing within South Africa.

\section{Regarding HIV prevention in the context of mental disorders:}

5. True (A) or false (B):

Individuals diagnosed with mental illness in South Africa have a level of knowledge of HIV prevention that is similar to that of the general population.

6. True (A) or false (B):

Standard HIV prevention messages and interventions may not be adequate for this group, as they have an increased risk of HIV infection compared with the general population.

Regarding the use of microscopy to diagnose a febrile HIVinfected infant:

7. True (A) or false (B):

High Candida fungal loads may lead to abnormal results of white cell count quantification in peripheral blood.

8. True (A) or false (B):

Peripheral blood smears may be useful in interpreting unusually rapid changes in full blood count parameters.

Regarding primary breast lymphoma in the context of HIV/AIDS:

9. Primary extranodal lymphoma is the most common presentation of nonHodgkin's lymphoma.

10. Non-Hodgkin's lymphoma is the most common AIDS-associated malignancy.
11. Which of the following are common presenting symptoms of nonHodgkin's lymphoma in HIV/AIDS:

A. Pain

B. Fever

C. Sweating and weight loss

D. All of the above.

Regarding invasive obstetric procedures in HIV-infected women:

12. Most current guidelines recommend that pregnant women with higher CD4 cell counts (e.g. >350 cells/ $\mu$ l) do not require any form of prophylaxis or therapy to prevent mother-to-child transmission of HIV before undergoing an invasive obstetric procedure such as amniocentesis.

13. If a woman is established on effective antiretroviral therapy and has an undetectable viral load, invasive obstetric procedures do not appear to significantly increase the risk of mother-to-child transmission of HIV.

14. In contexts where the invasive obstetric procedure is urgent and an HIVinfected woman has not received any form of antiretrovirals, initiation of therapy immediately before or after the procedure may still provide some measure of protection against HIV transmission.

\section{Regarding HIV prevention and treatment interventions for men} who have sex with men (MSM):

15. In terms of post-exposure prophylaxis, unprotected anal intercourse is a low-risk activity for HIV transmission, and it is appropriate to use 2-drug PEP.

16. There is evidence that pre-exposure prophylaxis using antiretroviral medications (PrEP) can help to protect MSM from sexually transmitted HIV infection, but adherence is an important consideration.

17. Anal intra-epithelial neoplasia (AIN) is recognised as an AIDS-related malignancy that is associated with the human papillomavirus.

18. Existing evidence suggests that the predominant subtype of HIV circulating in MSM in South Africa is subtype B (also the most common subtype among MSM in Europe and North America), while subtype C is more common in heterosexual populations in SA.

19. HIV prevention messages aimed solely at heterosexuals invariably include the risks associated with anal sex.

20. Recreational drugs can cause drug-drug reactions and side-effects in HIVpositive individuals taking antiretroviral medication. 